\title{
Curso virtual: educación STEM/STEAM, concepción e implementación. Experiencias de su ejecución con docentes costarricenses
}

\author{
Virtual course: STEM/STEAM education, conception and implementation. \\ Experiences of its execution with Costa Rican teachers
} Curso virtual: educação STEM/STEAM, concepção e implementação.
Experiencia de sua implementação com professores costarriquenhos

\author{
Marco Vinicio López-Gamboa \\ Universidad de Costa Rica \\ San José, Costa Rica \\ marcovinicio.lopez@ucr.ac.cr \\ (D) ORCID: https://orcid.org/0000-0003-4477-6487
}

Recibido - Received - Recebido: 30 / 06 / 2021 Corregido - Revised - Revisado: 05 / 09 / 2021 Aceptado - Accepted - Aprovado: 30 / 09 / 2021

DOI: https://doi.org/10.22458/ie.v23iespecial.3620

URL: https://revistas.uned.ac.cr/index.php/innovaciones/article/view/3620

\begin{abstract}
Resumen: Se presentan las experiencias durante un curso virtual sobre educación STEM/STEAM, dirigido al profesorado y funcionarios administrativos de diferentes contextos educativos, se desarrollaron contenidos como los modelos y los pasos para su implementación. Asimismo, sobresalen aspectos como el entorno virtual utilizado para su desarrollo y su respectiva interfaz; actividades de mediación pedagógica utilizadas, así como su evaluación. También se muestra parte de los productos realizados por las personas participantes del curso, en los que destacan limitaciones y abordajes, así como diseños de ambientes de aprendizaje para la implementación de la educación STEM/STEAM en sus correspondientes contextos educativos. Lo anterior, a partir de la investigación al implementar el curso virtual; pues el propósito principal de esta experiencia, fue dotar de herramientas y consideraciones para la implementación de este tipo de educación. Finalmente, destacar las limitaciones y los abordajes que proponen las personas participantes, así como las diferentes propuestas de proyectos de implementación de educación STEM/STEAM, en la cual destacan alcances, objetivos, metodologías y sus diferentes formas de evaluación.
\end{abstract}

Palabras clave: STEAM, pandemia, contexto, innovación educativa, formación profesional.

Summary: The experiences are presented during a virtual course on STEM/STEAM education, addressed to teachers and administrative officials from different educational contexts, contents such as models and steps for their implementation were developed. Likewise, aspects such as the virtual environment used for its development and its respective interface stand out, as well as pedagogical mediation activities used, as well as their evaluation. It also shows part of the products made by the participants of the course, which highlight limitations and approaches, as well as designs of learning environments for the implementation of STEM/STEAM education in their corresponding educational contexts. The above, based on the investigation when implementing the virtual course, since the main purpose of this experience was to provide tools and considerations for the implementation of this type of education. Finally, highlight the limitations and approaches proposed by the participants, as well as the different proposals for STEM/STEAM education implementation projects, in which the scope, objectives, methodologies and their different forms of evaluation stand out.

Key Words: STEAM, pandemic, context, educational innovation, professional training.

Resumo: Apresenta as experiencias durante um curso virtual sobre educação STEM/STEAM, para o corpo docente e funcionários administrativos de diversos contextos educacionais, no qual foi desenvolvido conteúdos como os modelos e passos para sua implementação. Também, destaca aspectos como o ambiente virtual usado para seu desenvolvimento e sua respectiva interface; atividades de mediação pedagógica utilizadas, y sua avaliação. Também mostra parte dos produtos realizados pelos participantes do curso, nos que se destacam as limitações e abordagens, como também projetos de ambientes de aprendizagem para a implementação STEM/STEAM em seus correspondentes contextos educacionais. O anterior, com base na pesquisa feita durante implementação do curso virtual; pois o principal objetivo 
desta experiencia, foi dotar de ferramentas e considerações para a implementação deste tipo de educação. Finalmente, cabe ressaltar as limitações e abordagens propostas pelos participantes, bem como as diversas propostas de projetos de implementação da educação STEM/STEAM, nas quais se destacam, escopos, objetivos, metodologias e suas diferentes formas de avaliação.

Palavras-chave: STEAM, pandemia, contexto, inovação educacional, formação profissional.

\section{INTRODUCCIÓN}

En la actualidad la educación STEM/STEAM ha incursionado en muchos contextos educativos de América Latina, primero para potenciar el interés de los estudiantes en carreras de la línea STEM, como: ingenierías, ciencias básicas y matemática; lo anterior con el fin de acrecentar las vocaciones por estas áreas de conocimiento (Perales-Palacios y Aguilera, 2020). Asimismo, como una potenciadora de estrategias didácticas activas, como el aprendizaje basado en problemas, el juego y demás, con lo cual los estudiantes, adquieren un rol más protagónico en sus procesos de enseñanza y aprendizaje; mientras los docentes se convierten en guías y facilitadores, en lugar de solo transmisores de conocimiento.

Por eso, la iniciativa del curso es dotar a las personas participantes, no solo de herramientas y consideraciones para implementar la educación STEM/STEAM en sus diferentes contextos; sino, también, mostrarles la concepción, los modelos de implementación y los pasos para su eventual desarrollo.

La sistematización de la experiencia, expone lo desarrollado en un curso virtual sobre educación STEM/ STEAM dirigido al profesorado y funcionarios administrativos de instituciones educativas, para su formación profesional. De ahí que un curso bajo esta modalidad, se acopla tanto a este aspecto como a la disposición de tiempo de las personas participantes.

Asimismo, la educación virtual también ha adquirido un importante papel en la actualidad, tanto para las personas estudiantes como para el profesorado en general, potenciada debido a la pandemia asociada a la COVID-19. Así pues, la necesidad de formación de personas docentes en educación STEM/STEAM, por medio de un curso virtual, no solo llega a fortalecer el conocimiento de estos por tal tendencia educativa; sino, los introduce en lo referente a los entornos virtuales de aprendizaje (EVA) y herramientas digitales para que las utilicen en sus respectivos contextos educativos.

La educación STEM es definida por Vásquez et al. (2013) como un acercamiento interdisciplinario al aprendizaje que remueve las barreras tradicionales de las cuatro disciplinas (Ciencias-TecnologíaIngeniería-Matemáticas) y las integra al mundo real con experiencias rigurosas y relevantes para los estudiantes. Asimismo, este tipo de educación, se ha convertido en el objeto de atención internacional para las reformas curriculares de la enseñanza de las ciencias, tecnología, ingeniería y matemáticas (Bogdan y Retana-Alvarado, 2021).

Por otro lado, se incorporó a este acrónimo la letra " $\mathrm{A}$ " correspondiente a las "Artes" o "the Arts", desde la perspectiva de las artes liberales de Yakman (2008), que además de las artes, promueve la participación de las ciencias sociales y otras áreas del conocimiento en la educación STEAM o CTIMA en español.

Dentro de las concepciones de la educación STEM/STEAM, se tiene que es una potenciadora de competencias científicas y tecnológicas, además de las habilidades del siglo XXI, como pensamiento crítico, sistémico, comunicación, colaboración, entre otros. Por lo general, el término más utilizado es STEAM, pero por el enfoque del artículo, utilizaremos "educación STEM/STEAM" en adelante, para hacer una mención y generalización de ambos acrónimos.

En lo que respecta a la forma de implementar este tipo de educación, The Arizona STEM Network led by Science Foundation and Maricopa County Education Service Agency (Red STEM de Arizona, la Fundación 
de Ciencia de Arizona y la Agencia de Servicios Educativos del Condado de Maricopa), propone cuatro modelos que se describen en la figura uno.

Figura 1. Modelos de implementación de educación STEM/STEAM
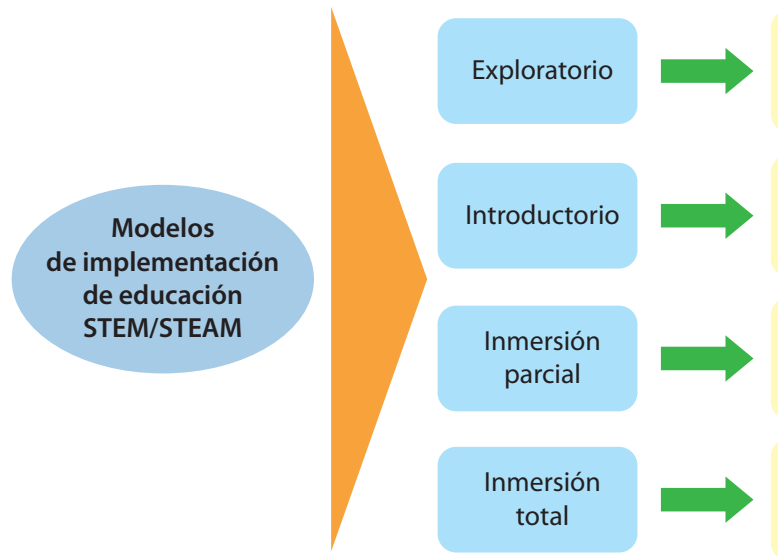

Desarrollo de actividades adicionales e independientes a la jornada ordinaria escolar, como los clubes de arte y robótics, etc.

Nota: adaptado de The Arizona STEM Network, 2017.

Como en todo proceso inicial para cualquier innovación educativa, lo recomendable es realizarlo de forma gradual, comenzar con el modelo exploratorio hasta eventualmente llegar al de inmersión total. Aunque dependerá del respectivo análisis de contexto que se realice en las instituciones educativas, así como, la disposición del recurso humano, infraestructura y equipamiento con la que estas cuenten. Además, de los objetivos de estas, en el sentido e interés que tengan; es decir, de repente la intención o contexto de una institución educativa en particular, sea solo desarrollar educación STEM/STEAM hasta el modelo introductorio; mientras para otra, sea el de inmersión total.

De todas formas, lo más elemental ante el desarrollo de cualquier iniciativa educativa, es la voluntad del personal y la capacidad de gestión institucional para llevarla a cabo, de ahí que se recomiendan los siguientes pasos, expuestos en la figura dos.

Figura 2. Pasos para la implementación de la educación STEM/STEAM

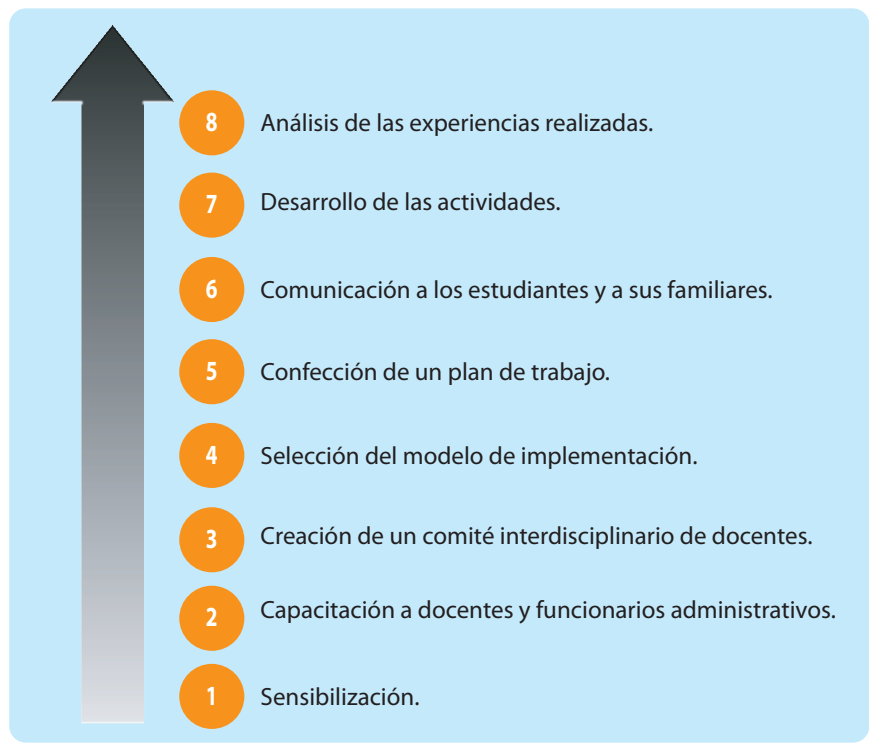

Fuente: elaboración propia, 2021. 
Previo al momento de implementar la educación STEM/STEAM en cualquier tipo de institución educativa, es importante considerar los pasos anteriores o al menos la mayoría, siempre en virtud de su respectivo contexto, que consisten en lo siguiente:

1. Sensibilización: en el sentido de presentar la idea de desarrollar la educación STEM/STEAM ante las autoridades educativas correspondientes.

2. Capacitación a docentes y funcionarios administrativos: para que conozcan en qué consiste este tipo de educación, explicar los modelos de implementación, pasos, estrategias didácticas, entre otros aspectos; pero sobre todo, darles a entender su articulación y los diferentes roles que pueden tomar.

3. Creación de un comité interdisciplinario de docentes: para que se encargue de realizar un diagnóstico de la disposición del cuerpo docente, infraestructura y equipamiento; asimismo de un análisis curricular e integración de los programas de estudio y la evaluación. Así como la confección de ambientes de aprendizaje (de ser necesarios), además de determinar cuáles actividades que se realizarán, las asignaturas en la que se van a desarrollar, entre otros aspectos.

4. Selección del modelo de implementación: según lo analizado y lo diagnosticado por el comité, en el paso tres, se define con cuál o en qué modelo debe o está la institución educativa, ya sea el exploratorio, el introductorio, la inmersión parcial o total.

5. Confección de un plan de trabajo: con los cronogramas de las actividades de educación STEM/ STEAM a realizar, ya sean trimestrales, semestrales o anuales, las personas participantes involucradas, las agendas de las reuniones del comité del paso tres. Así como su respectiva calendarización, para evitar choques con otras actividades y toda la comunidad de antemano conozca y se adecue a estas.

6. Comunicación a estudiantes y sus familiares: para explicarles en qué consiste la educación STEM/ STEAM y las diversas dinámicas de trabajo en clase, casa y evaluación.

7. Desarrollo de las actividades: ejecución de las actividades de educación STEM/STEAM en la asignatura o asignaturas que se hayan definido.

8. Análisis de las experiencias realizadas: de parte del comité establecido en el paso tres, para así determinar cuáles aspectos mejorar, cambiar; manejar un registro de evidencias. Además de generar un informe para exponer ante los cuerpos administrativos, docentes de la institución e incluso público en general de ser el caso.

Los pasos anteriores son una recomendación a considerar al momento de implementar la educación STEM/STEAM o cualquier innovación educativa. Estos no distan de muchos de los procesos usuales, los cuales se realizan en las instituciones educativas en sus diferentes planificaciones de tareas y proyectos. Además, de ser un rumbo para los funcionarios administrativos y docentes, que se desean introducir en este tipo de educación a nivel institucional.

Por otra parte, los EVA se pueden desarrollar por medio de diferentes formas, a partir de diversas modalidades de aprendizaje como el e-learning explicado por Prieto y Van Pol (2006), como la utilización de tecnologías digitales en la red. Además, del m-learning, que incorpora el uso de smartphones u otros dispositivos móviles como las tablet's, con o sin conexión a internet, de acuerdo con las respectivas apps que se estén usando.

Todo lo anterior, complementa el Conocimiento Tecnológico-Didáctico del Contenido (CTDC) de los profesores, expresado como la representación de los conceptos con el uso de la tecnología, a la vez que se integra con los contenidos de la respectiva disciplina y la didáctica (Mishra y Koehler, 2006). 


\section{DESARROLLO DE LA EXPERIENCIA}

Esta experiencia se desarrolló, bajo el marco de un curso 100\% virtual, Ilamado "Educación STEM/STEAM, concepción e implementación", en la plataforma Moodle, asincrónico en su mayoría, con una sesión sincrónica en la que se realizaron reflexiones finales, a través de Zoom. Se impartió en dos ocasiones, la primera durante abril y mayo, y la segunda entre junio y julio de 2020; contó con la participación de 40 personas, entre quienes destacan profesionales del sector educativo como orientadores, docentes de educación preescolar, primaria y secundaria. En esta última, la mayoría con una experiencia laboral que va desde un año hasta los 20 años. Para el caso de los profesores de secundaria, destacaban de áreas académicas por ejemplo Estudios Sociales, Matemáticas y Español; así como de especialidades técnicas como Mecánica Automotriz y Agroindustria.

Las personas participantes fueron invitadas a llevar el curso de forma gratuita, como parte del desarrollo investigativo y fomento de la educación STEM/STEAM por parte NeuroAula, división educativa de la empresa privada costarricense I.S. Corporación.

La experiencia se fundamenta en la investigación y la acción, descrita como ese proceso continuo del profesorado, que integra la reflexión y el trabajo intelectual en el análisis de sus experiencias educativas (Bauselas Herreras, 2004). Con ello se realiza un análisis cualitativo de la información obtenida durante el desarrollo de la experiencia; por medio de las diferentes actividades desarrolladas como tareas, foros y proyecto final, descritos en la tabla uno, así como un cuestionario diseñado en Google Forms, previamente validado por medio de un grupo piloto.

Se destacan los principales elementos de esta experiencia en las secciones: características del curso e interfaz del EVA, productos realizados por las personas participantes del curso y opiniones de las personas participantes del curso. Para posteriormente contrastarlos y obtener las respectivas síntesis y reflexiones finales en torno a estos.

\section{Características del curso e interfaz del EVA}

Dentro de las características de este curso virtual destacan:

- Duración: ocho semanas (40 horas en total), distribuidas en cinco horas semanales que el participante debe dedicar.

- Tipo: aprovechamiento.

- Dirigido: docentes preescolar, primaria, secundaria y universidad, así como funcionarios administrativos entre directores, coordinadores, asistentes de dirección, entre otros.

- Secciones del aula virtual:

- Bienvenida al curso.

- Módulos compuestos de clases semanales.

- Despedida.

Entre los contenidos del curso destacan:

- Netiqueta.

- Definición de educación STEM/STEAM.

- Actores principales del STEAM: funcionarios administrativos, docentes, estudiantes, comunidad y familia. 
- Modelos de implementación: exploratorio, introductorio, inmersión parcial e inmersión total.

- Pasos para la implementación de la educación STEM/STEAM.

- Estrategias didácticas: aprendizaje basado en problemas, proyectos, juego, entre otros.

- Ambientes de aprendizaje: aulas taller, colaboratorios, laboratorios: físicos, virtuales y remotos; entre otros.

- Habilidades del siglo XXI: pensamiento crítico, alfabetización digital, trabajo en equipo, entre otras.

Con respecto a la evaluación, tenía una nota mínima de aprobación de 80 sobre 100, cuyos rubros eran los siguientes: dos foros de discusión de $5 \%$ cada uno, cinco tareas de $10 \%$ cada una y un proyecto final de $50 \%$.

A continuación, en la tabla uno se describen algunos de los rubros o las actividades de mediación pedagógica presentados en la tabla.

TABLA 1

Algunas actividades de mediación pedagógica del curso virtual

\section{Actividad}

Foro de discusión 2: ¿qué impide que implemente la educación STEM/STEAM en mí institución educativa?

Tarea 2: planeamiento de clase que involucre la educación STEM/STEAM desde el enfoque de la asignatura que imparto.

Proyecto final.

\section{Descripción}

Consistía en que las personas participantes expresaran las diferentes circunstancias que notaran en sus instituciones, las cuales desde su perspectiva dificulten la aplicación de educación STEM/STEAM.

Trataba de que las personas participantes de forma individual realizaran un plan de clase, de máximo tres lecciones en las que implementara la educación STEM/ STEAM, contemplando aspectos interdisciplinares; además de describir las actividades que realizarían y la forma de evaluarlas.

Consistía en un trabajo grupal, en el cual las personas participantes confeccionaran una propuesta de proyecto que fomentara la educación STEM/STEAM, entre varias asignaturas. Asimismo, tenían que mostrar los recursos requeridos y las maneras en que se evaluarían las actividades propuestas.

Fuente: elaboración propia, 2021.

El curso se desarrolló en la plataforma digital de aprendizaje Moodle, pero puede implementarse en otras plataformas digitales para el aprendizaje como Schoology, Blackboard o similares. Debido a que se confeccionó de tal forma que lo que se requiera en el EVA se pueda insertar por medio de enlaces y videos; además, se puedan asignar tareas y foros con límites de tiempo, características que se pueden encontrar en estas otras plataformas. A continuación, en las figuras de la tres a la cinco se muestran unas capturas de imagen del entorno gráfico del EVA.

Figura 3. Sección de bienvenida del curso virtual

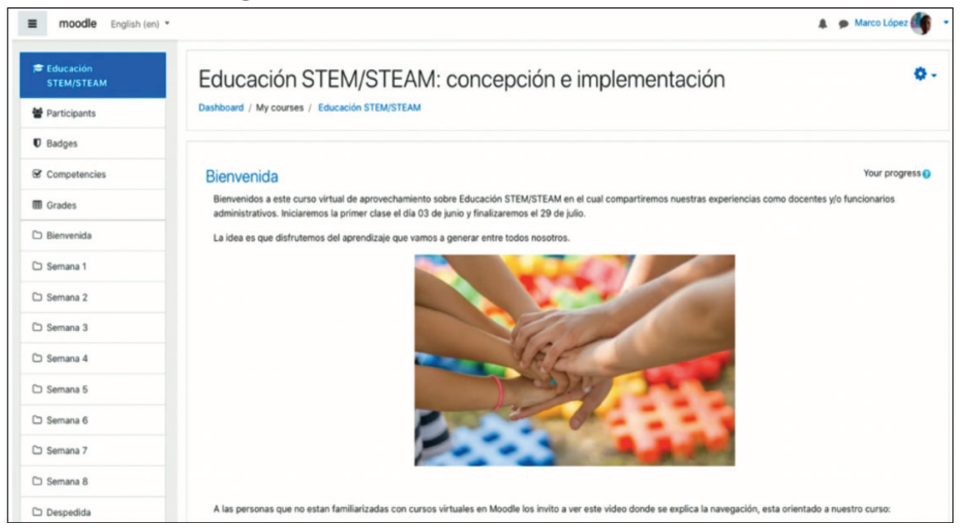

Fuente: Captura de pantalla curso Educación STEM/STEAM. 
En la figura tres se aprecia un mensaje de bienvenida dirigido a las personas participantes del curso, además de las fechas de inicio y cierre. Mientras que en la figura cuatro, se muestran algunos módulos semanales, además de otros elementos como: clases, foros y carpetas.

Figura 4. Algunos de los módulos semanales del curso virtual

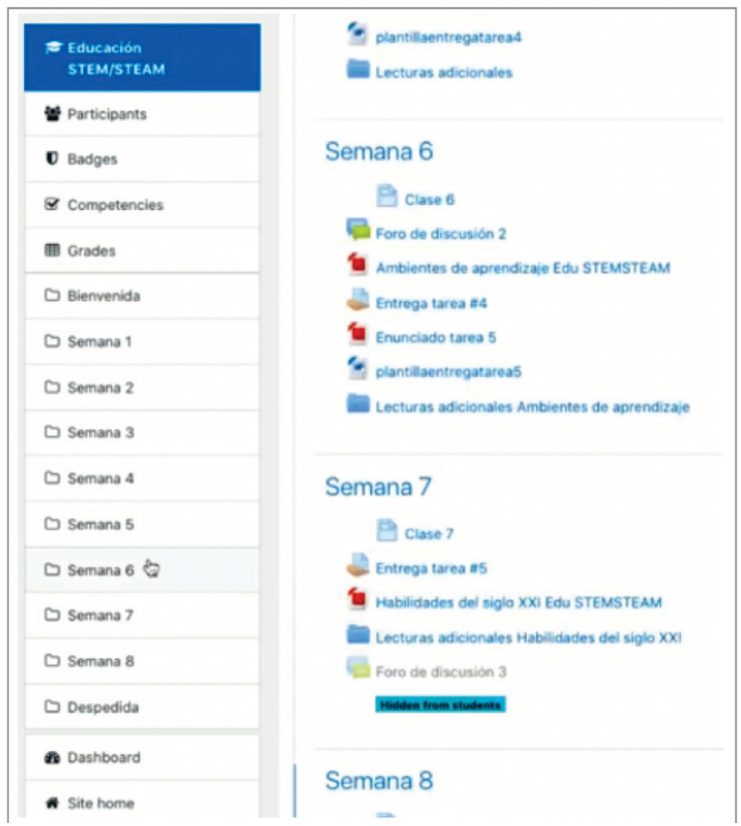

Fuente: Captura de pantalla curso Educación STEM/STEAM.

Las secciones de "Clase \#", están conformadas con las instrucciones respectivas a desarrollar durante la semana, así como los enlaces de los recursos y los foros a desarrollar; además, nociones teóricas para el desarrollo de la sección, como se aprecia en la figura cinco.

Figura 5. Algunos de elementos expuestos en las secciones "Clase \#"

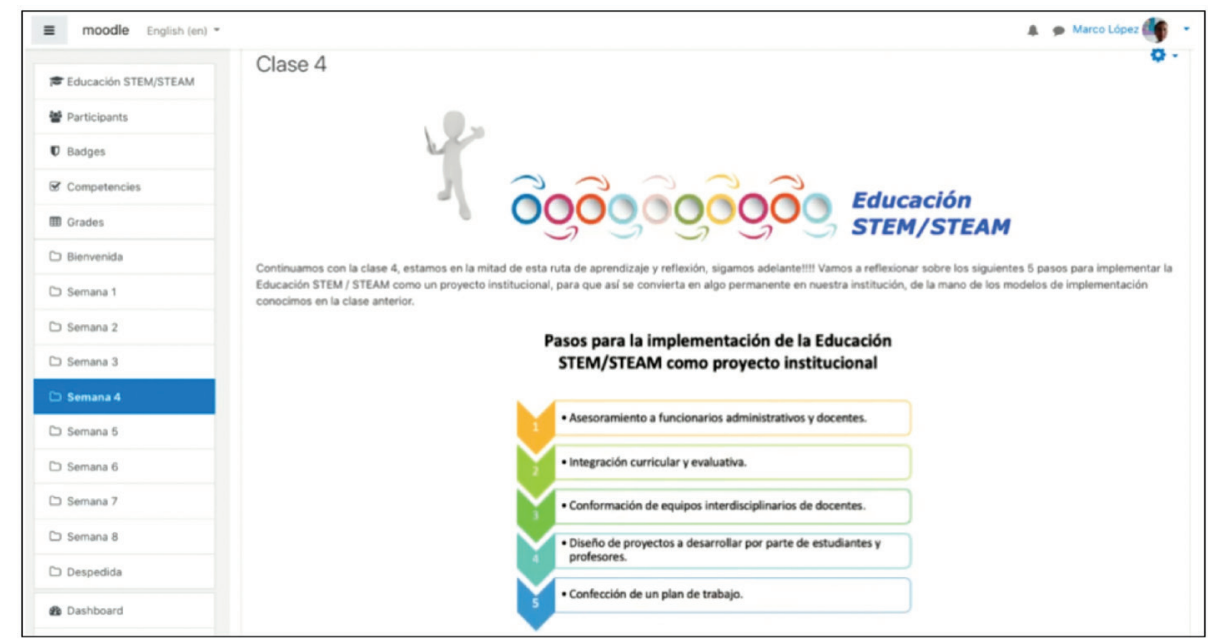

Fuente: Captura de pantalla curso Educación STEM/STEAM.

Para más detalles de la conformación y la estructura del curso, así como la descripción, sus bloques y más, en el siguiente código QR en la figura seis, se da acceso a un video que muestra diversos aspectos: 
Figura 6. Video de descripción de estructura del curso virtual

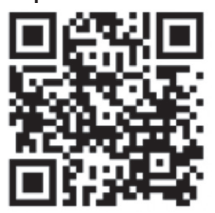

Fuente: López (2020).

\section{Productos realizados por las personas participantes del curso}

Las personas participantes del curso, realizaron diversas actividades de mediación pedagógica durante el desarrollo de este, en sus dos cohortes, como las indicadas en la tabla uno. A continuación, se presentan algunos de los productos asociados a estas actividades, diseñadas por las personas participantes, en el caso de la tarea tres, se solicitó que indicaran cinco limitaciones para implementar la educación STEM/ STEAM en su contexto educativo, así como su respectivo abordaje, en la tabla dos se presentan algunas expresadas por estas.

TABLA 2

Limitaciones y abordajes para la implementación de la educación STEM/STEAM

\begin{tabular}{|c|c|}
\hline Limitaciones & Abordaje \\
\hline \multicolumn{2}{|l|}{ Profesor de Ciencias Naturales (Secundaria) } \\
\hline $\begin{array}{l}\text { El apego a las maneras tradicionales de dar } \\
\text { clases. }\end{array}$ & $\begin{array}{l}\text { Se puede resolver organizando capacitaciones continuas en el uso de recursos tecno- } \\
\text { lógicos a los docentes de todas las asignaturas. }\end{array}$ \\
\hline $\begin{array}{l}\text { El considerar los recursos tecnológicos úni- } \\
\text { camente como elementos distractores. }\end{array}$ & $\begin{array}{l}\text { Creando una cultura de respeto y uso responsable en el estudiantado sobre el uso } \\
\text { de la tecnología; el saber limitar los tiempos de ocio y el uso correcto de netiqueta. }\end{array}$ \\
\hline $\begin{array}{l}\text { El relacionar los recursos tecnológicos úni- } \\
\text { camente con la enseñanza de la ciencia. }\end{array}$ & $\begin{array}{l}\text { Se puede abordar igualmente con el uso de capacitaciones sobre los distintos usos } \\
\text { diversos. }\end{array}$ \\
\hline $\begin{array}{l}\text { La poca disposición de recursos } \\
\text { tecnológicos. }\end{array}$ & $\begin{array}{l}\text { Crear planes de uso de los laboratorios y otros equipos de manera equitativa entre } \\
\text { el estudiantado. }\end{array}$ \\
\hline Los costos continuos de mantenimiento. & Abordaje mediante una cultura de uso responsable de los recursos tecnológicos. \\
\hline \multicolumn{2}{|c|}{ Profesora de Agropecuaria (Secundaria-Técnica) } \\
\hline Estancamiento de docentes. & Mayor capacitación a los docentes y ellos acepten actualizarse. \\
\hline Aprovechamiento de los recursos. & Aprovechar al máximo los recursos con los que cuenta la institución. \\
\hline Mayor participación de estudiantes. & Involucrar al estudiantado en programas de investigación e innovación. \\
\hline $\begin{array}{l}\text { Financiamiento de programas. } \\
\text { Infraestructura. }\end{array}$ & $\begin{array}{l}\text { Realizar alianzas estratégicas con empresas para programas de investigación. } \\
\text { Laboratorios aún no están listos para poder utilizarlos correctamente y explotar el } \\
\text { potencial de los mismos. }\end{array}$ \\
\hline \multicolumn{2}{|l|}{ Profesora de educación primaria } \\
\hline Espacio físico del aula. & $\begin{array}{l}\text { Solicitar a la administración del centro educativo que se mantenga al grupo a mi } \\
\text { cargo en una de las aulas más grandes de la institución. }\end{array}$ \\
\hline Mobiliario. & $\begin{array}{l}\text { Gestionar junto con los padres de familia donaciones de mobiliario escolar más acor- } \\
\text { des con el trabajo colaborativo. No usar negrita. }\end{array}$ \\
\hline Equipo de cómputo. & $\begin{array}{l}\text { Dentro de las mismas gestiones para que nos donen mobiliario, buscar la donación } \\
\text { de equipo de cómputo que pueda ser usado por los estudiantes. }\end{array}$ \\
\hline $\begin{array}{l}\text { Cobertura de internet. } \\
\text { Colaboración de otros docentes. }\end{array}$ & $\begin{array}{l}\text { Gestionar ante la Junta de Educación la mejora de la conexión a internet, el que haga } \\
\text { las solicitudes ante el ICE para que terminen conexión de la fibra óptica. } \\
\text { Motivarlos a conocer sobre la educación STEM/STEAM y compartir conmigo la } \\
\text { experiencia. Además, que tomen cursos como este. }\end{array}$ \\
\hline
\end{tabular}


En la tarea cinco, se solicitó la propuesta de diseño de un ambiente de aprendizaje, para el desarrollo de la respectiva asignatura que cada participante imparte, lo cual fomentará a la educación STEM/STEAM. Como parte de esta asignación debían entregar un diagrama en 2D o 3D del espacio propuesto, así como una explicación de cómo iban a desarrollar sus clases. A continuación, se presentan algunas de las entregas por parte de las personas participantes del curso virtual, así como su respectiva descripción por parte de cada una de ellas.

Figura 7. Propuesta de ambiente de aprendizaje por parte de un profesor de Estudios Sociales (secundaria)

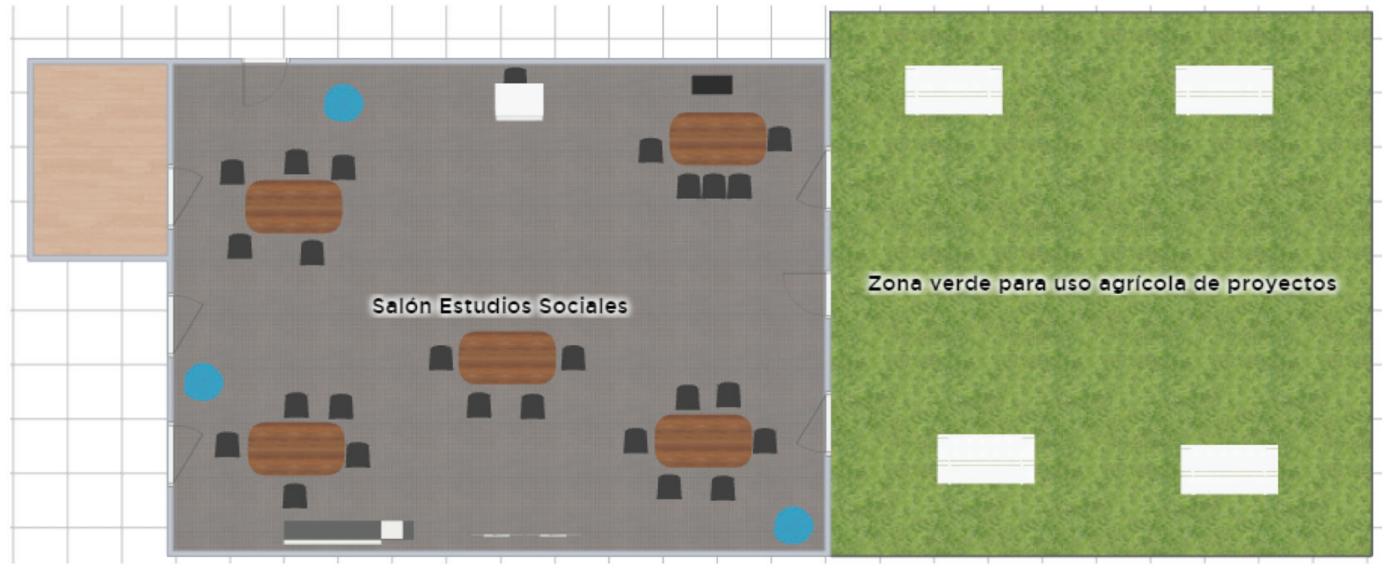

Fuente: Captura de pantalla curso Educación STEM/STEAM.

El desarrollo de las clases en un ambiente como el ilustrado en el diseño se puede llevar a cabo de diferentes formas, esto debido a que los contenidos de Estudios Sociales abarcan diversas temáticas. Por ejemplo, cabe destacar que el salón principal posee mesas para trabajos colaborativos; pues como bien se sabe el trabajo en equipo en nuestra actualidad viene a ser parte fundamental de la formación de los futuros profesionales. Asimismo, se incentivará lo que es la investigación mediante el uso de herramientas como tablets, Chrome books, móviles y otros.

Figura 8. Propuesta de ambiente de aprendizaje por parte de una profesora de Agropecuaria (Secundaria-Técnica)

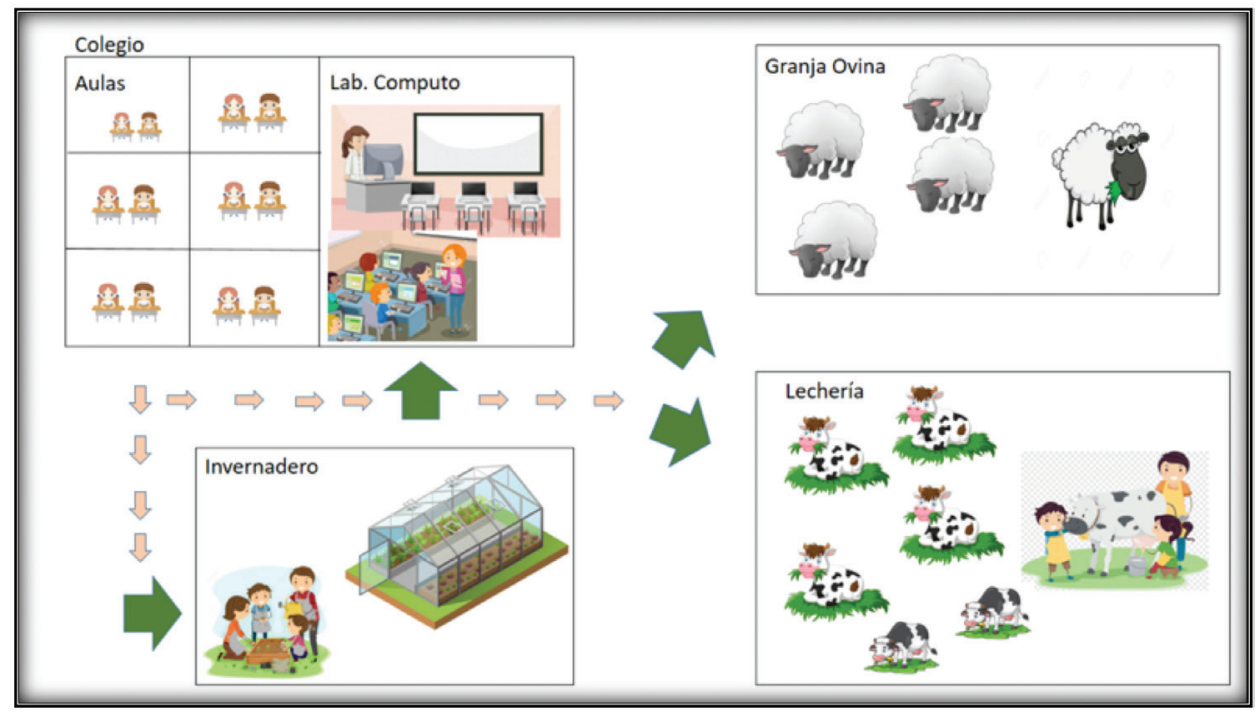

Fuente: Captura de pantalla curso Educación STEM/STEAM. 


\section{Descripción}

Los estudiantes reciben sus clases teóricas en sus respectivas aulas en el colegio, pero luego se dirigirían a la práctica de estas, ya sea en la granja ovina, lechería o invernadero, esto dependería del plan de estudio.

Los estudiantes en la asignatura de agropecuaria incorporan las matemáticas cuando deben sacar pesos en los ovinos; pues detallan en una hoja de Excel los promedios de peso en que se ubica la granja ovina, al igual en la lechería con las pesas de leche y estos datos los toman y los aplican en el laboratorio de cómputo. Esto va de la mano ya que ellos utilizan las computadoras del laboratorio para llevar los registros de los animales o las cosechas del invernadero.

Figura 9. Propuesta de ambiente de aprendizaje por parte de una orientadora (Secundaria-Técnica)

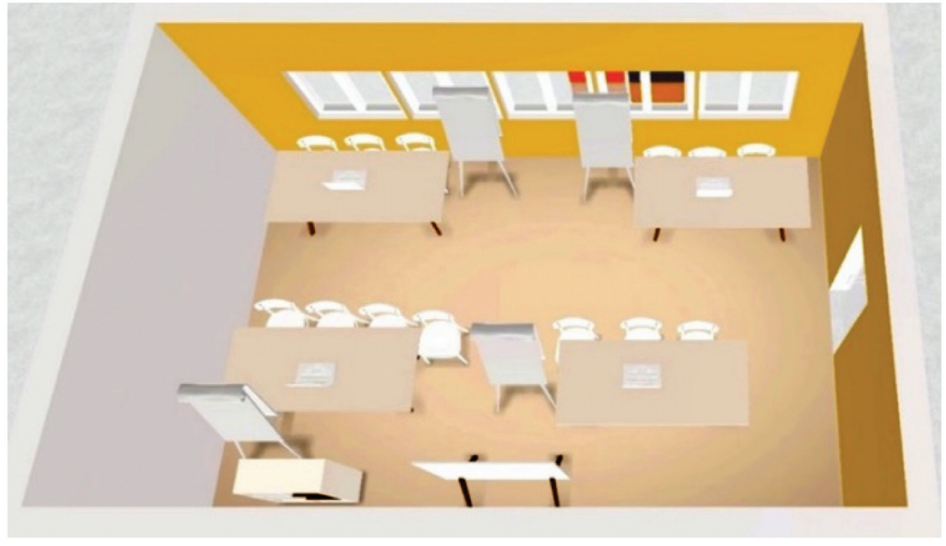

Fuente: Captura de pantalla curso Educación STEM/STEAM.

\section{Descripción}

Orientación no brinda lecciones en sí, sino imparte talleres compuestos por varias sesiones, las cuales no tienen un espacio definido, esto varía según la temática que se desea impartir.

Generalmente, se trabaja en espacios como aulas sin pupitres o en salones amplios. Por lo tanto, el diseño anterior es un salón grande que cuenta con mesas amplias y sillas, lo cual permite a los estudiantes trabajar en subgrupos y luego poder compartir lo trabajado con los demás compañeros.

Figura 10. Propuesta de ambiente de aprendizaje por parte de una docente de Primaria

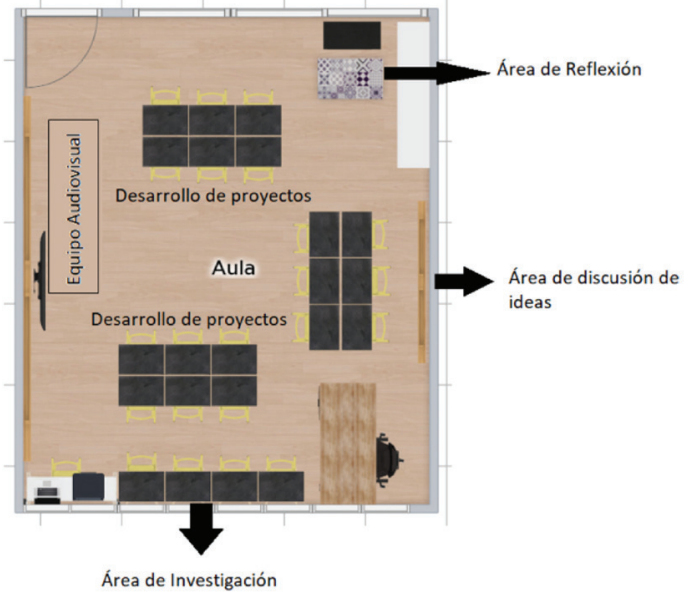

Fuente: Captura de pantalla curso Educación STEM/STEAM. 


\section{Descripción}

El Área de Investigación sería destinada para que diferentes grupos de estudiantes se desplacen a esta zona para buscar información en la Web, la cual les pueda ser de utilidad para sus proyectos. Se cuenta con una impresora para imprimir aquella información que sea necesaria.

El Área de Discusión de Ideas cuenta con una pizarra acrílica, la cual puede utilizarse por los grupos de estudiantes para plasmar sus ideas, rutas de trabajo, avances de proyectos.

El Área de Reflexión cuenta con una alfombra y un mueble con hojas, cartulinas, marcadores, para que allí los grupos de trabajo puedan sentarse a reflexionar sobre el avance de sus proyectos, sobre cómo mejorarlos o resolver un problema que se les está presentando.

Mientras que en la tarea dos, se asignó la formulación de un planeamiento didáctico que involucrará la implementación de la educación STEM/STEAM desde su respectiva asignatura. En la figura once, se presenta un código QR que da acceso a un archivo en formato ".pdf", en el cual se muestran tres planeamientos, presentados por un profesor de Ciencias Naturales de secundaria, una docente de primaria y una profesora de inglés de secundaria respectivamente:

Figura 11. Algunos planeamientos que involucran educación STEM/STEAM

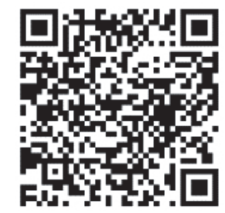

Fuente: Elaboración propia.

Para el proyecto final, único trabajo grupal, se solicitó la confección de una propuesta de proyecto que fomentará la educación STEM/STEAM entre varias asignaturas, para así potenciar la interdisciplinariedad y el trabajo en equipo entre el profesorado, representado por las personas participantes del curso. Para ello se resaltan elementos, tales como: limitaciones, alcances, objetivos, descripción del proyecto, metodología, así como las reflexiones finales, plasmadas en función de diferentes contextos y recursos disponible para la eventual ejecución de la propuesta. La figura doce corresponde a un código QR, con el cual se puede apreciar un documento en formato ".pdf" con tres de estas propuestas de proyectos.

Figura 12. Algunos proyectos finales que involucran educación STEM/STEAM

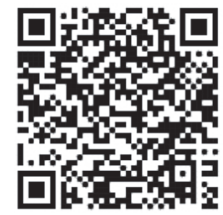

Fuente: Elaboración propia.

En estas propuestas, se describe la interdisciplinariedad entre asignaturas académicas como técnicas, entre las que destacan Estudios Sociales, Ciencias, Agropecuaria, Dibujo técnico, entre otros; de la mano de la integración de habilidades para el siglo XXI como el trabajo en equipo, pensamiento crítico e investigación. A su vez, se desarrollan diferentes formas de evaluación, tanto formativas como sumativas, para cada asignatura como de forma conjunta, entre las propuestas, se resaltan los modelos exploratorios y de inmersión parcial como se puede observar en la figura doce, todos centrados en la puesta de las personas estudiantes como actores principales de sus procesos de enseñanza y aprendizaje. Además, los respectos cronogramas de ejecución, algunos yendo desde los dos meses hasta los seis meses, todo en función de sus objetivos de trabajo. 


\section{Opiniones de las personas participantes del curso}

En esta sección destacan algunas opiniones de las personas participantes del curso virtual, las cuales se obtuvieron por medio de un cuestionario realizado en Google Forms, que se muestra en el siguiente código QR en la figura trece. En este se les solicitaba información variada, desde sus años experiencia, asignatura que imparten, modelo de implementación que consideraban más sencillo de aplicar en su institución, hasta detalles intrínsecos del curso virtual, como si le recomendaría a otros colegas, la mediación de la persona facilitadora del curso; asimismo recomendaciones para futuras ejecuciones del curso, por motivos de espacio, no se presentan todas las opiniones, así como otras informaciones y datos obtenidos.

Figura 13. Cuestionario aplicado a las personas participantes del curso virtual para obtener sus opiniones

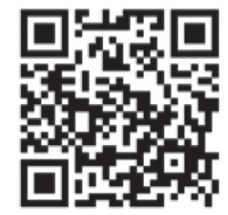

Fuente: elaboación propia.

A continuación, se presentan algunas de las opiniones:

¿Cuál de los modelos de implementación estudiados en el curso, considera más fácil de aplicar en la institución educativa en la que labora? ¿Por qué?

Profesora de Turismo (Secundaria-Técnica):

Exploratorio. Porque mis clases lo permitirían debido a que son especialidad técnica.

Profesora de educación Preescolar:

Exploratorio. Integrando poco a poco con los contenidos curriculares

Profesor de Ciencias Naturales (Secundaria):

Introductorio. Porque los estudiantes trabajan por proyectos para dar respuesta a un problema de la sociedad de forma interdisciplinaria y los resultados se exponen a la comunidad estudiantil, mucho trabajo en equipo, se fomenta la creatividad.

Profesora de Matemática (Secundaria):

Inmersión parcial. Porque creo que es un modelo adecuado para iniciar, implementando proyectos en donde se integren tres o cuatro materias de la malla curricular.

Profesor de Ciencias Naturales (Secundaria):

Inmersión total. En la modalidad científica el enfoque STEAM-STEM es vivido diariamente a través de laboratorios virtuales, presenciales, investigaciones dirigidas, entre otras, actividades que obedecen a un currículo diseñado para favorecer el aprendizaje por indagación, resolución de problemas, por pares, clase invertida. En los colegios públicos se puede trabajar el STEAM-STEM bajo modelos introductorios, realizando actividades en el aula que no serían parte de las pruebas nacionales o el currículo vigente. 
¿Considera que la educación STEM/STEAM potencia las habilidades para el siglo XXI? Sí o No. ¿Por qué?

Profesora de Agroindustria (Secundaria-Técnica):

Sí. Se enseña para los trabajos del futuro.

Profesor de Mecánica Automotriz (Secundaria-Técnica):

Sí. Relaciona especialidades y disciplinas pilares del desarrollo actual.

Orientadora:

Sí. Es una metodología que se aplica a las nuevas generaciones y son necesarias.

Profesora de educación Primaria:

Sí. Permite que el estudiante tome decisiones, trabaje en equipo, potencie la comunicación.

Profesora de Sicología (Secundaria):

Sí. Esta educación STEM/STEAM toma en cuenta cada habilidad y capacidad de los estudiantes, donde el docente es el guía. Engloba todas las habilidades en un ser humano. Ejemplo la capacidad de ser crítico, creativo, innovador, trabajo en equipo, la comunicación entre otras.

Destacar que todas las personas participantes del curso respondieron afirmativamente a la pregunta.

¿Qué recomendaciones o sugerencias daría usted para mejorar el curso?

Profesora de educación Preescolar:

Considero que se pueden implementar más intervenciones virtuales, tipo conferencia, esto puede favorecer la interacción entre los participantes.

Profesora de Estudios Sociales (Secundaria):

Estuvo bien, tal vez lo sentí cargado en algún momento, pero es por la situación particular en la que tuvimos que llevar el curso donde se nos ha duplicado el trabajo desde casa. Pero en general bastante bien estuvo el curso.

Especialista en Educación Ambiental (programas de educación no formal):

Salir del esquema formal del MEP. Darle espacio a la creatividad en la entrega de tareas, des-estructurar la visión de un documento en el que todos deben presentar el mismo formato. Además, la plataforma me costó tiempo aprender a usarla y entenderla.

Profesor de Ciencias Naturales (Secundaria):

Estimular más foro de participación. Una parte informal. Para conectar con la dimensión afectiva de los participantes y lograr mayor interés.

Profesor de Inglés (Secundaria):

Que sea más interactivo, poner en práctica las estrategias y un poco de mayor información sobre los modelos de implementación.

Es importante mencionar que, aunque se expongan las opiniones de la misma asignatura, estas corresponden a personas participantes diferentes. 


\section{SIINTESIS Y REFLEXIONES FINALES}

Se plasma la versatilidad de la educación virtual como recurso didáctico, que de la mano de diferentes herramientas tecnológicas como la plataforma Moodle y las actividades de mediación pedagógica como las expuestas en la tabla uno. Con ello se promueve en las personas participantes, no solo el intercambio de ideas y dudas sobre esta tendencia educativa; sino, sobre la marcha, conocieran y desarrollarán experiencias, sobre cómo es llevar un curso en línea, así como otras herramientas digitales, como las utilizadas para diseñar las propuestas de ambiente de aprendizajes, representados en las figuras 7, 8, 9, y 10.

En cuanto a la formación profesional de las personas participantes, estas adquirieron conocimientos sobre la educación STEM/STEAM, de la mano de un fortalecimiento de su CTDC, debido al desarrollo del curso virtual y los diferentes requerimientos tecnológicos que este solicitaba, como lo visto en las $7,8,9$, y 10 . Por otra parte, el intercambio de opiniones, a través de los foros de discusión y el proyecto final descritos en la tabla uno, deriva una fuente de aprendizaje para las personas participantes del curso virtual. Todo lo anterior, fundamentado en los principios del Conectivismo de Siemens (2005) de que el aprendizaje puede residir en dispositivos no humanos y el aprendizaje y el conocimiento dependen de la diversidad de las opiniones, respectivamente.

Sobre el curso virtual en sí, es importante destacar algunos de los aspectos obtenidos por medio de las tareas y demás actividades de mediación, como lo expuesto en la tabla dos, en la que se destacaron diferentes limitaciones y sus respectivos abordajes sobre la implementación de la educación STEM/ STEAM o cualquier innovación educativa en sus contextos educativos, los cuales coincidieron en aspectos como la anuencia de los profesores a colaborar o implementar actividades nuevas, debido a que están apegados a las maneras convencionales de impartir clases, su poca anuencia a utilizar recursos TIC, así como detalles de infraestructura.

De ahí que es importante la capacitación y la utilización de los recursos ya existentes, como lo mencionan en los respectivos abordajes; además, de no asociar que para aplicar educación STEM/STEAM, se requiere de equipo de tecnología digital o laboratorios de última generación.

En lo que respecta a las limitaciones y los abordajes expresados por el profesorado en la tabla dos, destacan la tendencia al desarrollo de metodologías tradicionales y la carencia de recursos, como principales obstáculos para una eventual implementación de la educación STEM/STEAM, lo cual pone en evidencia la tendencia de algunas personas docentes por no salir de una zona de confort ya estructurada y definida; de la mano con la creencia de que para desarrollar actividades nuevas en los procesos de enseñanza y aprendizaje, es indispensable contar con recursos, tales como: nueva infraestructura física y equipos nuevos.

Por otro lado, lo correspondiente a los abordajes, destacan la capacitación continua, aprovechamiento de los recursos con lo que ya la institución cuente, la generación de alianzas para la obtención de recursos varios, como el fomento de programas de investigación, recursos tecnológicos e infraestructura.

Con lo anterior, se destaca la importancia de la formación profesional en este tipo de educación, de la mano, con el correspondiente análisis de contexto, para el aprovechamiento máximo de los recursos con lo que cuente la institución y determinar los que se necesiten a futuro, para ello se priorizan los pasos del dos al cuatro expuestos en la figura dos.

En cuanto a los proyectos finales, destacar que algunos grupos, estuvieron conformados por personas docentes, de diferentes niveles educativos, como primaria y secundaria; asimismo, de diferentes instituciones educativas, lo que dio una variedad de criterios a la confección de las propuestas. En cuanto a los modelos de implementación sugeridos en las propuestas destacó en su mayoría el de inmersión parcial; pues como se describe en la figura uno, este puede desarrollarse de la mano de algunos contenidos y 
rubros de evaluación que seleccionen en la institución; por lo tanto, es el más acorde al contexto educativo de la mayoría de las personas participantes. En otras palabras, seleccionaban dos o tres contenidos o un contenido base, y desarrollaban la propuesta en torno a esa selección, como se detalla en la figura doce.

Finalmente, resaltar las opiniones expresadas por las personas participantes del curso por medio del cuestionario que se aprecia en la figura trece; las cuales son una fuente importante de retroalimentación y aprendizaje, para futuras ediciones de este curso virtual, como el aumento del componente sincrónico, para así compartir y obtener más reflexiones y aprendizajes con las personas participantes del curso, escuchar sus inquietudes inmediatas. Además de sus experiencias en sus distintos contextos educativos, así se genera una interacción en tiempo real entre la persona facilitadora con las personas participantes.

Por otra parte, es importante promover más la participación en los foros, para así aumentar la interacción no solo entre la persona facilitadora del curso; sino entre las personas facilitadoras, para evitar que estos se conviertan en espacios en los cuales estas exponen sus ideas o respuestas a premisas, desaprovechando el intercambio de ideas y la generación del debate y reflexión en torno a estas.

\section{AGRADECIMIENTOS}

A I.S. Corporación que a través de su división educativa NeuroAula propició la ejecución del curso y facilitó el entorno virtual de aprendizaje.

\section{REFERENCIAS}

Bausela Herreras, E. (2004). La docencia a través de la investigación-acción. Revista lberoamericana De Educación, 35(1):1-9. Recuperado desde: https://doi.org/10.35362/rie3512871

Bogdan, R. y Retana-Alvarado, D. A. (2021). Mejora de las concepciones de maestros en formación de la educación STEM. Revista Iberoamericana de Educación, 87(1): 15-33. Recuperado desde: https://doi.org/10.35362/rie8714538

López, M. [Marco López]. (2020, setiembre 05). Descripción de curso Virtual en Moodle 1. [Archivo de video]. Recuperado desde: https://youtu.be/lv515DhLRh8

Mishra, P. \& Koehler, M. (2006). Technological Pedagogical Content Knowledge: A Framework for Teacher Knowledge. Teacher College Record, 106(6):1017-1054. Recuperado desde: http://one2oneheights.pbworks.com/f/MISHRA_PUNYA.pdf

Perales-Palacios, F. J. y Aguilera, D. (2020). Ciencia-Tecnología-Sociedad vs. STEM: ¿evolución, revolución o disyunción? Ápice. Revista de Educación Científica, 4(1):1-15. Recuperado desde: https://doi.org/10.17979/arec.2020.4.1.5826

Prieto, D. y Pol, P. (2006). e-Learning comunicación y educación. El diálogo continúa en el ciberespacio. (1a Ed.). San José, Costa Rica: Radio Nederland Training Centre.

The Arizona STEM Network. (2017). STEM Inmersion Guide. Recuperado desde: http://stemguide.sfaz. org/stem-implementation-guide/

Siemens, G. (2005). Connectivism: A Learning Theory for the Digital Age. International Journal of Instructional Technology \& Distance Learning, 2:1-9. Recuperado desde: https://bit.ly/3BfdRJj

Vásquez, J., Sneider, C. \& Comer, M. (2013). STEM Lesson Essentials, Grades 3-8 Integrating Science, Technology, Engineering and Mathematics. (1a Ed.). USA: Heinemann.

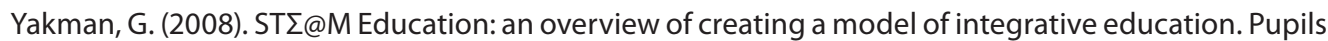
Attitudes Towards Technology 2008 Annual Proceedings. Netherlands. Recuperado desde: https://bit.ly/2FSnFkQ 